\title{
CORRESPONDENCE
}

\section{Nosocomial catheter-associated Flavobacterium odoratum bacteraemia in cancer patients}

Flavobacterium odoratum is known to be a soil and plant saprophyte [1]. While there are numerous reports of life-threatening infections (septicaemia, meningitis) caused by $F$ meningosepticum only two due to $F$. odoratum have been reported [2]. The virulence of $F$. odoratum is, therefore, uncertain [1-3].

Here we report four cases of catheter-associated polymicrobial bacteraemia caused by $F$. odoratum and another organism (Table 1). Two of four cases were caused by $F$. odoratum and $C$. albicans and two by $F$. odoratum and coagulase-negative staphylococci (CNS). All strains were obtained by routine blood culture and identified with the Vitek Jr. system (Vitek Systems, bioMerieux). Two patients were hospitalised on the leukaemia ward, one in another ward for lymphomas and one in a ward for patients with solid tumours, all in a single cancer institution (National Cancer Institute). Fingerprinting was not done, as the first case occurred in 1992, the second in 1994, the third in 1995 and the fourth in early 1997. All strains were isolated from blood cultures. Three of four patients were neutropenic, and in three, breakthrough bacteraemia appeared despite prophylaxis with ofloxacin (200 mg twice daily) even though all strains of $F$. odoratum were susceptible to ofloxacin. All four bacteraemias were catheter-associated, and after removal of the catheter, symptoms (i.e., fever) disappeared. Three patients were treated with antimicrobial agents and one with catheter removed only. In the two cases in which CNS were isolated, vancomycin was used in monotherapy, even though vancomycin has no in-vitro activity against Flavobacterium spp. In patient no. 1 only, amikacin and ceftriaxone were administered in addition to catheter removal.

Patients details are outlined in Table 1. All strains except one were susceptible to most antimicrobial agents tested (gentamicin, amikacin, ofloxacin, ciprofloxacin, netilmicin, tobramycin, azlocillin). Only isolates from patient no. 4 were resistant to thirdgeneration cephalosporins.

In conclusion, $F$ odoratum may cause systemic infection as a breakthrough bacteraemia; however, its virulence seems to be relatively low compared with that of $F$. meningosepticum, as all patients were cured following catheter removal.

\section{S. Spanik, J. Trupl and V. Krcmery} Department of Medicine, Microbiology and Pharmacology, National Cancer Institute and St Elizabeth Cancer Institute, Heydukova 10,812 50 Bratislava, Slovak Republic.

\section{References}

1. Sader HS, Jones RN, Pfaller MA. Relapse of catheter-related Flavobacterium meningosepticum bacteremia demonstrated by DNA macrorestriction analysis. Clin Infect Dis 1995; 21: 9971000.

2. Hsueh PR. Bacteremic necrotizing fasciitis due to Flavobacterium odoratum. Clin Infect Dis 1995; 21: 1337-1338.

3. Macfarlane DE, Baum-Thureen P, Crandon I. Flavobacterium odoratum ventriculitis treated with intraventricular cefotaxime. $J$ Infect 1985; 11: 233-238.

Table 1. Four cases of $F$. odoratum bacteraemia in cancer patients

\begin{tabular}{|c|c|c|c|c|c|c|c|c|c|c|}
\hline $\begin{array}{l}\text { Case } \\
\text { no. }\end{array}$ & Age/sex & $\begin{array}{l}\text { Underlying } \\
\text { disease }\end{array}$ & $\begin{array}{l}\text { No. of } \\
\text { positive } \\
\text { blood } \\
\text { cultures }\end{array}$ & $\begin{array}{c}\text { Neutropenia } \\
<500 \text { at } \\
\text { time of } \\
\text { bacteraemia }\end{array}$ & $\begin{array}{l}\text { Central } \\
\text { venous } \\
\text { catheter } \\
\text { insertion }\end{array}$ & $\begin{array}{l}\text { Source } \\
\text { of bacter- } \\
\text { aemia }\end{array}$ & Prophylaxis & Polymicrobial & Therapy & $\begin{array}{l}\text { Out- } \\
\text { come }\end{array}$ \\
\hline 1 & $42 / \mathrm{M}$ & NHL & 4 & + & + & $\begin{array}{l}\text { Infected } \\
\text { catheter }\end{array}$ & Ofloxacin & $\begin{array}{l}+ \text { Candida } \\
\text { spp. }\end{array}$ & $\begin{array}{c}\text { Amikacin }+ \\
\text { ceftriaxone } \\
+ \text { catheter } \\
\text { removal }\end{array}$ & Cured \\
\hline 2 & $60 / \mathrm{F}$ & $\begin{array}{l}\text { Solid tumour } \\
\text { (gastric Ia) }\end{array}$ & 3 & - & + & $\begin{array}{l}\text { Infected } \\
\text { catheter }\end{array}$ & None & $\begin{array}{l}+ \text { Candida } \\
\text { spp. }\end{array}$ & $\begin{array}{l}\text { Catheter } \\
\text { removal }\end{array}$ & Cured \\
\hline 3 & $31 / \mathrm{M}$ & AML & 5 & + & + & $\begin{array}{l}\text { Infected } \\
\text { catheter }\end{array}$ & Ofloxacin & + CNS & $\begin{array}{l}\text { Catheter } \\
\text { removal } \\
\text { vancomycin } \\
1 \mathrm{~g} \mathrm{bd} 7 \\
\text { days }\end{array}$ & Cured \\
\hline 4 & $20 / F$ & AML & 3 & + & + & $\begin{array}{l}\text { Infected } \\
\text { catheter }\end{array}$ & Ofloxacin & $+\mathrm{CNS}$ & $\begin{array}{l}\text { Catheter } \\
\text { removal } \\
\text { vancomycin } \\
1 \mathrm{~g} \mathrm{bd} 7 \\
\text { days }\end{array}$ & Cured \\
\hline
\end{tabular}

